\title{
The development of stereoscopic warehouse stacker control system based on motion controller
}

\author{
Xuan Sun ${ }^{*}$, Zhedong Ma, Zhiyong Wang and Changsheng Ai \\ School of Mechanical Engineering, University of Jinan, Jinan, China
}

\begin{abstract}
In order to meet the requirement of high efficiency, high accuracy, high reliability and high expansibility of stacker. The stacker control system based on motion controller is developed,which uses digital control technology of $\mathrm{NC}$ machine tools for reference and adopts the half closed loop control system of motion controller and servo drive system to realize high efficiency, high precision and high reliable motion control of stacker.Using the network interface of controller interact with the logistics system server, storage management, computer information management system of ERP, AGV car, palletizing robot and other equipment.Make it becomes the organic part of the logistics system, which provides a good solution to improve the level of enterprise logistics automation.
\end{abstract}

\section{Introduction}

Stereoscopic warehouse is an important component of the intelligent logistics system. It is the key link of the warehousing and transportation system. It is the result of the development of logistics and storage system and the progress of information technology [1].Now the warehouse has been developed integrated software product that consists of the mechanical and electrical combination, combination of strong current and weak current control, automatic feeding system, automatic sorting system, automatic control system, AGV transport system, automatic stacking stack system and host computer control software [2].Compared with the traditional platform warehouse and floor warehouse, it has incomparable advantages.It is the key node in the intelligent factory and Internet of things system.

\section{Requirement analysis of mechanical structure and motion control of stacker}

The mechanical of the stacker is composed of operating mechanism, lifting mechanism, extension fork mechanism, loading platform, frame and various safety protection devices [3].The structure is shown in Fig.1.

In order to complete the incoming and outbound operations of the goods, the stacker needs to meet the design standards : Need the direction of three degrees of freedom, which is moving in horizontal direction, realize the column positioning of the goods listed in warehouse; The vertical movement of the cargo platform to realize the goods layer positioning in warehouse;Bidirectional telescopic movement of goods fork achieves the access to the goods.It has the safety protection device for ensuring the safety of the stacker body and the operation person. There are chain breaking protection mechanism, overload protection device and limit protection device for each movement direction. The structural and mechanical properties of stacker meet the principles of strength, stiffness and reliability principle. The weight of each part should be reduced to minimize the impact of inertial impact on the acceleration, braking and operation of the stacker.At the same time ,manufacturing costs can also be reduced [4].

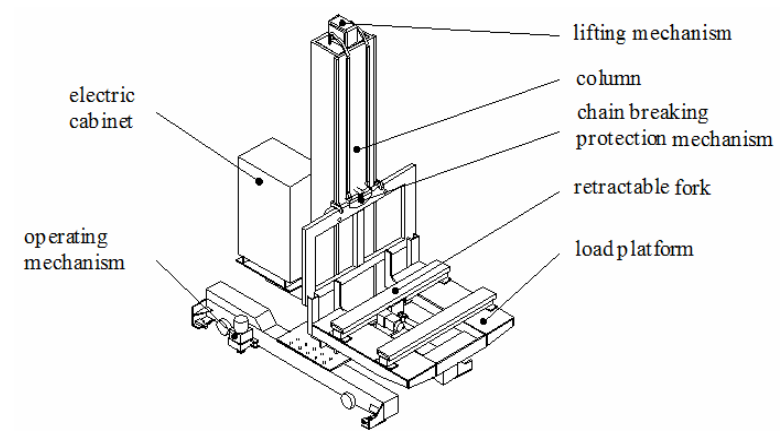

Fig. 1. Stacker structure

\subsection{Operating mechanism}

The major function is servo driver inside the electrical cabinet drive the servo motor that is mounted on the lower cross member of stacker.The gear on the motor output shaft meshes with the rack on the floor rail so that the stacker can move horizontally in order to realize the column positioning of the goods in storage.A walking wheel is arranged on one side of the lower cross beam, and a guide wheel is arranged on the other side, which plays a good guiding role in the smooth operation of the stacker. When the overload and stall conditions, servo driver will execute the overload protection, the motor will perform brake.

Corresponding author: me_sunx@ujn.edu.cn 


\subsection{Lifting mechanism}

The lifting mechanism is the mechanism that realizes the vertical lifting and power transmission.Because of the heavy load of the stacker, the counterweight mechanism which consists of reduction box and the counterweight block is designed to balance the load of the loading platform.Lifting mechanism adopts chain drive has the advantages of stable transmission, accurate average drive ratio, large transmission power and high overload capacity.The servo motor is mounted on the top of the column to drive the gear reducer to rotate. It is connected with the counterweight block in the column by the chain transmission, so as to pull the truck in the vertical direction.

\subsection{Fork part}

In order to improve the ground utilization ratio of stereoscopic warehouse and the utilization ratio of stacker, so as to reduce the number of stacker used in the same area, it is decided to adopt double extension fork.The mechanism is shown in Fig.2.The double extension fork adopts three level linear expansion mechanism, which is composed of drive motor, reducer, coupling, upper fork, middle fork, lower fork, chain wheel and transmission device [5].

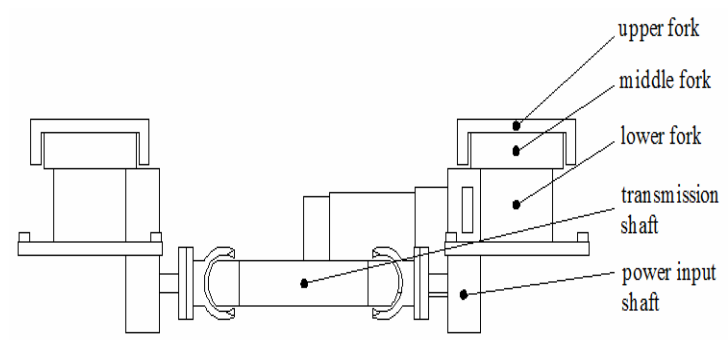

Fig. 2. Fork structure

\subsection{Cargo platform}

The cargo platform is an important goods bearing unit is mainly composed of a guide wheel, wheel frame, truck body and a guide seat, which is provided with a double extension fork and anti chain breaking device mechanism [6].Under the condition of meeting the strength and stiffness, on both sides of the ribs are opening with circular holes as far as possible to reduce its quality and effectively reduce the vertical motor dragging weight.The guiding mechanism can ensure the stable movement of the cargo platform in the vertical direction of the column, reduce the friction resistance and improve the positioning accuracy of the cargo table movement.

\section{Analysis of reliability and safety requirements for stacker crane}

Any hidden danger of insecurity is likely to cause incalculable losses to enterprises and users,so take the necessary protective measures and safety is very important.

\subsection{Limit position protection}

In the horizontal direction of the stacking machine, not only the zero limit switch is set, but also the limit position is set at both ends of the guide rail.The zero limit switch is arranged in the vertical direction, and the limit switch is also arranged to prevent the loading platform from moving beyond the safe stroke, and the falling prevention mechanism is arranged to prevent the accident of the motor from being broken and the chain breaking [7].

\subsection{Software protection}

In the stacker control software, the fork can perform the soft protection of the extended and retracted movements after the stacker is moving in a horizontal and vertical direction to a safe position. At the same time, the stacker can move horizontally and vertically when the fork moves to a safe position, which effectively prevents the collision between the stacker and the warehouse shelf.

\subsection{Power-off protection}

In case of accidental power failure, each axis servo motor has brake function effectively prevent the loss of power to each shaft accidents. This function makes each axis keep its original state to prevent the stacker goods falling and protect the personal safety of the operator.

\section{Stacker control system design}

\subsection{Stacker work flow analysis}

In stereoscopic warehouse, Stacker as an important out / storage of goods equipment that equivalent to the arms in a stereoscopic warehouse.It needs to complete the operation of warehousing and outbound operations.

\subsubsection{Warehousing operation}

After the stacker detects the arrival of the AGV, the stacker performs the action to read the tag information on the tray, reads the information to the stacker controller and displays it on the display screen.According to the rules of the nearest and uniform, the stereoscopic warehouse management system automatically assigns the pallet goods a target position. When the stacker performs the insertion and removal of the goods, it sends the completion notification to the host computer. The host computer tells AGV to leave.The pallet load is placed in the warehouse.If you read the electronic tag on the tray error, the system will automatically alert the alarm information.Waiting for the completion of manual processing, and then perform the appropriate action. 


\subsubsection{Outbound operation}

Warehouse has two kinds of outbound form,which is delivery of cargo from storage by position or type.The specific process is to enter the type of goods needed to be shipped out in the upper computer, and then inquire about the quantity or type of the goods stored in the warehouse,enter the quantity or type needed to be shipped out of the warehouse.The upper computer will send out the warehouse command to the stacker according to the FIFO rule, and the stacker will perform the corresponding action.

\subsection{Design of control hardware}

The warehouse control system hardware consists of six axis motion controller GUS, demonstrator, servo motor, servo driver, limit switch, origin switch, RFID electronic label reader, barcode scanner, outbound PC etc [8].Hardware diagram of stacker control system,as shown in Fig.3.

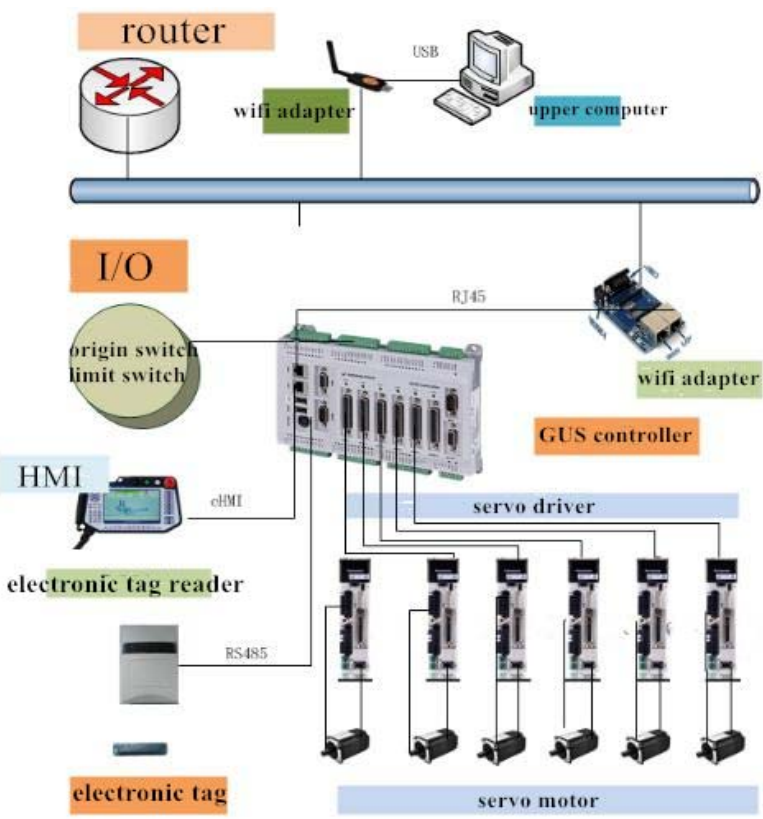

Fig. 2. Hardware composition of control system

The system uses the integrated motion controller as the core of the control system of the stereoscopic warehouse.It is connected with each axis servo control system through its axis interface. Receive user commands from the keyboard and mouse through the PS/2 interface.Various sensor signals and alarm signals are received through the input/output interfaces.The graphical interface program is displayed by the demonstrator and interacts with the user to return the result of the operation [6].

\subsection{Design of control software}

The automatic warehouse control system software is divided into eight functional modules:

- Return to zero: When the system is switched on, the stacker performs a zero return operation to establish a warehouse coordinate system to determine the distance of movement to each position;

- Back to standby point;

- The motion control of loading and unloading dispatch of the warehouse: automatically /manually loading and unloading dispatch of the warehouse;

- Back to standby point;

- Operation information management;

- Inventory information management:Users can view the current usage of warehouse, inventory model, storage time, storage amount in the warehouse browsing function in real time.The user can modify the stock information of the position in the software, and transmit the information to the stereoscopic warehouse stacker, which is convenient for users to view the current information in real time.

- Historical data:Users can view the software login, information status, login time.You can check the number of outbound and outgoing time of a certain model according to the time period.You can also check the delivery time and quantity of all the models in a period of time;

- System debugging: The parameters such as the zero return speed, the running speed, the position of the standby point and the extending distance of the fork are set up for each axle of the stacker When the whole system is debugged on-line. The user can operate the axes manually;

- System operation parameter setting:Set the stacker's zero return speed and acceleration, manual and automatic warehousing speed and acceleration, fork speed, acceleration and other parameters in order to achieve the smooth running of each axis of the whole system;

- Fault alarm and diagnosis: In the course of stacker operation, the corresponding warning information will be displayed on the handheld display device when the axle overload and servo alarm are failed.The occurrence of major errors also need to stop stacker. This method facilitates the operators to find the cause of the trouble and to eliminate the trouble in time.These alarms mainly including servo driver alarm, communication failure alarm, abnormal shutdown alarm, command execution failed alarm.

According to the functional requirements of the management system, the function diagram of the software of the stereoscopic warehouse management system is designed, as shown in Fig.4.

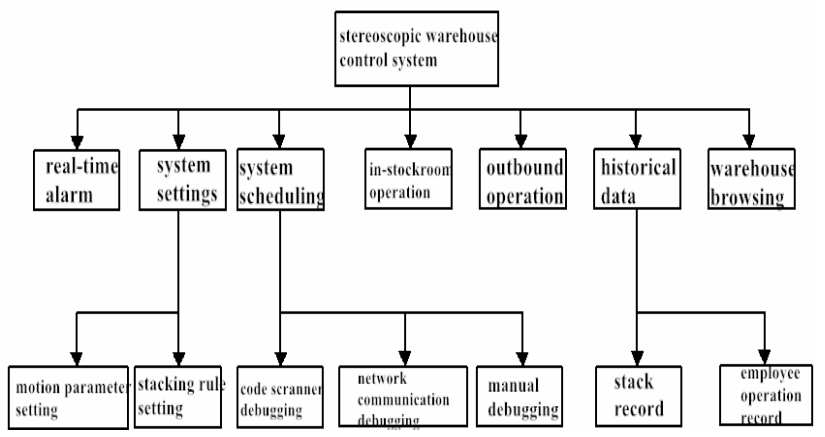

Fig. 4. Management system software structure 


\subsection{Control system speed and position control}

The motion of each axis of the control system is driven by a servo driver, servo motor drives the corresponding shaft movement, and the speed control sets corresponding parameters through the display screen. The movement of each axis is controlled by acceleration and deceleration. The advantage of this method is that the system runs smoothly, the impact load is smaller, the torque is large, the position control is accurate, and the mechanical characteristics are hard.

In the direction of movement of the $\mathrm{X}$ axis:At first, the acceleration was accelerated at $100 \mathrm{~mm} / \mathrm{s} 2$, uniform motion after speed up to $21 \mathrm{~m} / \mathrm{min}$, in the target position still has one position distances, with $100 \mathrm{~mm} / \mathrm{s}^{2}$ acceleration deceleration to the target position.In the direction of $\mathrm{Z}$ axis movement, there are two cases of loading and no-load in the loading platform.Two speed parameters are set for the two cases respectively.

At no-load, the $Z$ axis at first to $120 \mathrm{~mm} / \mathrm{s}^{2}$ acceleration operation, uniform operation after accelerated to $6 \mathrm{~m} / \mathrm{min}$.At a relatively short distance from the target position, slow down at $80 \mathrm{~mm} / \mathrm{s}^{2}$ acceleration to target position.At load, the $\mathrm{Z}$ axis at first to $80 \mathrm{~mm} / \mathrm{s}^{2}$ acceleration operation, uniform operation after accelerated to $5.5 \mathrm{~m} / \mathrm{min}$. At a relatively short distance from the target position, slow down at $80 \mathrm{~mm} / \mathrm{s}^{2}$ acceleration to target position. The stacker speed curve is shown in Fig.5.

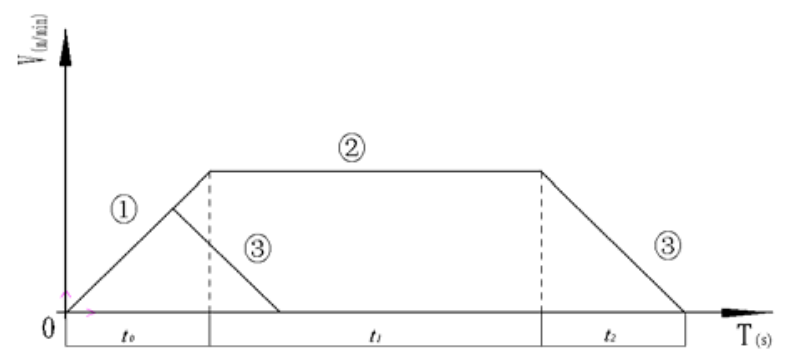

Fig. 5. Stacker operating speed curve

The warehouse uses a layout of the unit goods format, which requires an accurate location for each cell.There are three common positioning methods in stereoscopic warehouse, they are location of target address, location of laser range and location of pulse count [8].The position setting value of the actuator can be adjusted by man-machine interface in the position control process of the axle positioning, and then modified in the program.

The servo motor drives the stacker movement and converts the circumferential motion into linear motion through the rack and pinion mechanism. The position information of the actuator is fed back to the GUS motion controller via an encoder of the motor.The motion controller calculates the motor speed periodically according to the setting value and the actual value.The value is output to the servo drive via the pulse output port to form a half closed loop control that improves positioning accuracy [5].The stereo storage system uses servo motors with encoders. After practical testing, the positioning accuracy is $1 \mathrm{~mm}$, which can achieve the positioning function well and meet the requirements of the positioning accuracy of the system.

\section{Stacker software function module}

\subsection{Return reference point function module}

The incremental encoder is arranged on the servo motor of the stacker crane of the stereoscopic warehouse. This device has the advantages of high precision and good thermal stability, but the disadvantage that the zero point and the absolute position of the reference point can not be recorded after the power failure is taken. This requires each time you start the machine, you need to go back to the reference point and establish a position coordinate system to ensure the accuracy of the position when the goods are loading and unloading dispatch of the warehouse.

According to the system accuracy requirements $(1 \mathrm{~mm})$ and the actual situation of the system hardware, the system uses the Home switch to search the reference point.The origin search principle is shown in Fig.6.

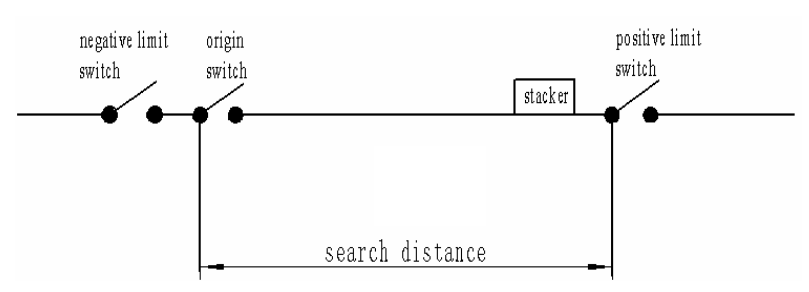

Fig. 6. Origin search principle

\subsection{Online automatic storage module}

When the AGV of the welding machine reaches the storage turntable and stops smoothly, the AGV sends in place signals to the storage management server through the router.The server reads the current state of the stacker after receiving the information.If the current stacker is idle, the management computer sends the storage operation instructions to the stacker controller, and then the stacker starts the automatic storage process.

\subsection{Manual storage function module}

Companies need to put some of the less commonly used or backlog of goods into the designated positions, or because of other reasons need to specify warehousing positions, so the need to develop manual warehousing functions to put the small volume goods in warehouse.In the manual storage case, the user need to manually enter the amount,type and target position. The controller checks the parameters entered by the user.If the parameter is valid, the stacker performs warehousing operations and puts the goods in the target position.After completion the storage, the stacker will return to standby and wait for the next order. 


\section{Conclusions}

In order to adapt to the rapid development of intelligent logistics industry, the stacker control system to carry out the study play an important role in the modern logistics management system, which can meet the automatic stereo warehouse to high speed, heavy load, high precision, high reliability, complex system development urgent needs. The system uses GUS embedded motion controller and servo system, the controller software development platform of OTOSTUDIO has rich library functions and powerful instruction systems.Developed a set of automated warehouse control system with high positioning accuracy, high efficiency of storage and delivery, stable and reliable operation. Combine it with computer software, which promotes the improvement on the control system of the logistics industry.After the actual operation test, the whole system has good coordination, reliable operation, accurate movement and high efficiency. It meets the requirements of each function and performance index of the stacker.

\section{References}

1. Riccardo Manzini, Mauro Gamberi. Design and control of an AS/RS. Int J Adv Manuf Technol. 2006, 11(28): 766-774.

2. Yasukawa, Machiko, Takaki, Masayoshi, et al. A study on slip and fall of stacked cargoes in automated warehouse.Structural and Construction Engineering, 2017, 82(732): 183-192.

3. Colla V, Nastasi G, Matarese N, et al. Ga-based solutions comparison for storage strategies optimization for an automated warehouse.Intelligent Systems Design and Applications, 2009. ISDA'09. Ninth International Conference on. IEEE, 2009: 976-981.

4. Eldemir F, Graves R J, Malmborg C J. New cycle time and space estimation models for automated storage and retrieval system conceptualization. International Journal of Production Research, 2004, 42(22):4767-4783.

5. Salah B, Janeh O, Noche B, et al. Design and simulation based validation of the control architecture of a stacker crane based on an innovative wire-driven robot. Robotics and Computer-Integrated Manufacturing, 2017, 44: 117128.

6. Kolla S, Michaloski J, Rippey W. Evaluation of component-based reconfigurable machine controllers.Automation Congress, 2002 Proceedings of the 5th Biannual World. IEEE, 2002, 14: 625-630.

7. Eldemir F, Graves R J, Malmborg C J. A comparison of alternative conceptualizing tools for automated storage and retrieval systems. International journal of production research, 2003, 41(18): 4517-4539.

8. Piasecki D. Warehouse Management Systems: Is It Worth the Trouble. World Trade, 2004, 17: 60-69. 